\section{THE CLINICAL BURDEN OF SEVERE SPORTS INJURIES IN ENGLAND AND WALES}

${ }^{1}$ Madeleine Davies, ${ }^{2}$ Tom Lawrence, ${ }^{2}$ Antoinette Edwards, ${ }^{2}$ Fiona Lecky, ${ }^{1}$ Carly McKay, ${ }^{1}$ Keith Stokes, 'Sean Williams. ${ }^{1}$ University of Bath, Bath, UK; ${ }^{2}$ Trauma Audit Research Network, Manchester, UK

\subsection{6/bjsports-2021-IOC.266}

Background Severe sports injury has substantial individual and societal health and financial implications. There has been limited study of the number, type, and clinical burden of severe injuries. Trauma research networks, such as the Trauma Audit Research Network (TARN) in England and Wales, may facilitate estimating the burden of severe sports injury.

Objective To estimate the number of severe sport-related injuries treated in hospitals in England and Wales between January 2012 and December 2017, and identify the 10 main contributing sports.

Design Five-year retrospective study.

Setting English and Welsh hospitals.

Patients All patients whose injury mechanism was indicated as sport, or whose incident description field featured one of 62 sporting activities, and qualified for inclusion in the TARN database. Inclusion criteria are: transfers or direct admissions whose inpatient stay is 3 days or more, admissions to High Dependency Areas and mortality after admission.

Assessment of Risk Factors Hospital-recorded age, sex and sporting activity at the time of injury.

Main Outcome Measurements A severe (TARN-recorded) injury, during in-game sporting activity. Extracted data included treatment duration, injury characteristics and sport code.

Results There were 15,799 sports injuries between 2012 and 2017. In 2012 there were 2,087 injuries (13.3\% of incidents), and by 2017 there were 2,906 (18.6\% of incidents). Patients were on average $37.7( \pm 19.5)$ years, and 6,396 (40.5\%) were female. The average length of hospital stay was 9.5 days (SD \pm 15.6 , range 1 to 738). Horse-related activities accounted for 5,585 of injuries, followed by football (soccer) with 1,439 injuries, motor racing $(\mathrm{n}=938)$, cycling $(\mathrm{n}=917)$, motocross $(n=826)$, off-road cycling $(n=669)$, rugby $(n=660)$, trampolining $(n=620)$, running $(n=501)$, and skiing $(n=326)$.

Conclusions Horse-related, football, motor racing and cycling injuries presented with the most injuries, and should be the focus of prevention efforts. Further work will examine sports participation data, and quantify severe injury risks associated with sports participation.

\section{INJURY RATES AND MECHANISMS OF INJURY IN FEMALE HIGH SCHOOL RUGBY}

${ }^{1}$ Isla Shill, ${ }^{1}$ Amanda M Black, ${ }^{1}$ Stacy Sick, ${ }^{1,2}$ Ash Kolstad, ${ }^{1,3}$ Luz Palacios-Derflingher, 1,2,4,6,7 Kathryn Schneider, ${ }^{1,2,3,5,8}$ Brent E Hagel, 1,2,3,4,5,8 Carolyn Emery. ${ }^{1}$ Sport Injury Prevention Research Centre, University of Calgary, Calgary, Canada; ${ }^{2}$ Alberta Children's Hospital Research Institute, Cumming School of Medicine, University of Calgary, Calgary, Canada; ${ }^{3}$ Department of Community Health Sciences, Cumming School of Medicine, University of Calgary, Calgary, Canada; ${ }^{4}$ Hotchkiss Brain Institute, Cumming School of Medicine, University of Calgary, Calgary, Canada; ${ }^{5}$ Department of Paediatrics, Cumming School of Medicine, University of Calgary, Calgary, Canada; ${ }^{6}$ Acute Sport Concussion Clinic, Sport Medicine Centre, University of Calgary, Calgary, Canada; ' Evidence Sport and Spinal Therapy, Calgary, Canada; ${ }^{8} \mathrm{O}^{\prime}$ Brien Institute for Public Health, Cumming School of Medicine, University of Calgary, Calgary, Canada

$10.1136 /$ bjsports-2021-IOC.267
Background In Canada, unlike many countries, youth rugby players often have their first exposure to the sport in high school (ages 15-16). There are few studies examining injuries in female high school rugby union.

Objective To describe injury rates and mechanisms among females participating in high school rugby union.

Design Prospective cohort study.

Setting Rugby pitches (Calgary, Canada).

Participants Female high school rugby players (ages 15-18) participating in 2018 (7 teams, $\mathrm{n}=214$ ) and 2019 (7 teams, $\mathrm{n}=207$ ) seasons.

Assessment of Risk Factors Mechanism of injury was recorded by team designates on an electronic injury report form, validated by a certified athletic therapist.

Main Outcome Measurements Training and match injuries were identified by a team designate or study therapist if the player 1) required medical attention, 2) was unable to complete the session, and/or 3) unable to participate in activity for $\geq$ one day.

Results There were 155 match $[93.7$ injuries/1000-matchhours (95\%CI, 78.6-111.7)] and 85 training injuries [5.3 injuries/1000-training-hours (95\%CI，4.0-6.9)] across two years of injury surveillance. Match injuries most commonly occurred while tackling [62 injuries (40\%) 37.5 injuries/ 1000-match-hours (95\% CI, 27.1-51.8), being tackled [47 injuries (30\%), 28.4 injuries/1000-match-hours (95\%CI, 20.339.8)], and during a ruck/maul [12 injuries (8\%), 7.3 injuries/1000-match-hours]. Training injuries most commonly occurred while tackling [20 injuries (24\%), 1.2 injuries/1000training-hours $(95 \% \mathrm{CI}, 0.7-2,4)]$, being tackled [17 injuries (20\%), 1.1 injuries/1000-training-hours (95\%CI，0.7-1.7)], and running [9 injuries (11\%), 0.6 injuries/1000-traininghours $(95 \% \mathrm{CI}, 0.3-1.0)]$.

Conclusions Tackling was identified as the most common mechanism of injury among female high school rugby players, with the highest rates in the active tackler during matches. Safe tackling interventions are an ideal primary prevention target to reduce the risk of injury within this population.

\section{SURVEILLANCE: INJURY INCIDENCE AND INJURY PATTERNS IN PROFESSIONAL FOOTBALL PLAYERS REGISTERED IN EGYPTIAN FOOTBALL LEAGUE}

Mohamed Yahia. Professor and Head of Orthopedic Department Al-Azhar University, Zahraa University Hospital, Cairo, Egypt

10.1136/bjsports-2021-IOC.268

Background Injury surveillance is an important pre-requisite for injury prevention planning. There is lack of information about injuries, rates, patterns and the procedures used for injury prevention of football players in the professional Egyptian soccer league.

Objective To record and describe the injury incidence and characteristics in male professional football participating in Egyptian football league by using a club based injury surveillance system.

Design Prospective study, where a random of 6 governors among 27 different governors existing in Egypt were selected and including all participating with man first team at footbal Egyptian league.

Setting Any injury detected for athletes in the first male team of professional football Egyptian league that were registered in 